Página inicial: 231 - Página final: 254

Tipo de artículo: de investigación

\title{
Hacia una perspectiva clínica psicodinámica de la intervención de las adicciones.
}

Toward a psycho-dynamic clinical perspective of addiction intervention.

Recibido: febrero de 2015 Revisado: octubre de 2015 Aceptado: noviembre 20 de 2015

Por: Francisco J. Arias $Z^{1}$ y Jorge Enrique Correa Uribe ${ }^{2}$.

\section{Resumen.}

El trabajo que se presenta a continuación muestra resultados de investigación sobre intervención del consumo de drogas desde una de las perspectivas de la psicología; nombrada como psicología dinámica que toma tanto de Freud como de post freudianos y que son retomados por muchos de los autores de que trata el presente escrito; el cual pretende también contribuir en la construcción de una guía clínica para la intervención de las adicciones.

\section{Palabras claves.}

Clínica, intervención, psicodinámica, consumo de sustancias

\section{Abstract.}

The work presented below introduces the results of research on drug consumption intervention from one of the perspectives of psychology; known as dynamic psychology that takes both from Freud and the post-Freudian, and which are taken up by many of the authors about the present writing; which aims to also contribute to the construction of a clinical guide for the intervention of addictions.

\section{Key words.}

Clinical; Intervention; Psycho-dynamic; and Substance consumption.

\footnotetext{
${ }^{1}$ Psicólogo, Magister en psicología, Especialista Contextualización Psicosocial del Crimen. Estudios clínicos y sociales en Psicologia (linea adicciones) de la USB. Medellin, Colombia. Contacto: francisco.arias@usbmed.edu. co; fjaz67@gmail.com

${ }^{2}$ Psicólogo, especialista en clínica con niños con énfasis en psicoanálisis. Estudios clínicos y sociales en Psicología (línea adicciones) de la USB. Contacto: jcorreau@une.net.co
} 


\section{Introducción.}

"La clínica de las toxicomanías no admite moldes colocados de manera apresurada sobre el material clínico, sino la necesidad de inventar modos de escuchar”. (Djambolakdjian, 2007, Pag.162).

El trabajo siguió entonces la lógica de la Investigación Documental la cual hace referencia al hecho de que el investigador adquiere la información por medio de documentos ya existentes, etc., incluido en ello, textos impresos como en medios electrónicos. En este tipo de estudio, junto con la importante labor de ordenar y analizar la información ya existente, la investigación no se limita a un trabajo descriptivo, sino que avanza a sistematizaciones y articulaciones entre el material recogido, que posibilitan nuevos conocimientos.

En este caso en particular, este estudio, que se inscribe en el contexto general de la Investigación Documental, corresponde a lo llamado Estado del Arte. Tipo de investigación que tiene por objeto, identificar, recuperar, reunir y organizar una vasta información, sobre un determinado tema. Como Estado del Arte, corresponde también a una de las modalidades de "Investigación de la Investigación", dado que busca sistematizar los trabajos realizados dentro de un área, llama la atención sobre los cambios más importantes, los enfoques y métodos presentes, destaca las relevancias, redundancias y vacíos dentro de tema (Hoyos Botero , 2000; CCI-FIUC, 2008).

Este texto recoge información en fuentes secundarias de lo producido en el contexto local, nacional e internacional y que fuera rastreada desde los centros de documentación y bases de datos de instituciones académicas de la región. El objetivo central estuvo dirigido hacia el conocimiento construidos y contenido en los documentos que han trabajado en esta línea de indagación y con ello la pretensión de buscar aportes con miras al diseño de una guía clínica para la intervención del consumo de sustancias psicoactivas desde una perspectiva psicodinámica. Se tuvieron en cuenta para el cumplimiento del objetivo, trabajos de grado, artículos de revistas físicas o electrónicas y libros y se realizaron búsquedas tanto en bibliotecas universitarias donde hubiese facultades de psicología locales, así como por intermedio de las bases de datos desde y con las cuales cuenta la Universidad de San Buenaventura.

\section{Metodología.}

El artículo da cuenta entonces de una Investigación de tipo documental, para lo cual se seleccionaron las "unidades de trabajo" siguiendo ciertas rutas como que los documentos reportasen preferiblemente informes de investigación y que tuviesen desarrollos relacionados con la intervención del consumo de sustancias psicoactivas y que fueran abordados desde una perspectiva dinámica de la psicología. Es de anotar que para la recolección y organización de la información, se adaptaron las fichas propias para el registro de información propuesta por Galeano E, 2008); una ficha descriptiva y otra de contenido para para consignar la información. Es de anotar que el trabajo se desarrolló atendiendo a una serie de Fases: en la primera se hizo contacto con fuentes secundarias, discusiones y hallazgos de pistas generales para la definición de los ítems, en la segunda se construyó la propuesta que contuvo los ítems que orientaron luego la pesquisa, selección de las estrategias de búsqueda y la revisión-adaptación de la ficha y los procedimientos de recolección, ya en la tercera fase se hizo la recolección, organización y sistematización de la información 
(primeras categorias) que incluyo primer y segundo contacto con las fuentes secundarias, ya para la cuarta y última fase se hace descripción según recategorización y elaboración del informe con resultados; es de anotar que las fases no se dieron de manera consecutiva sino más bien de forma paralela. Luego de la recolección de datos en las respectivas fichas, estas fueron sometidas a un proceso de evaluación para constatar que los datos fuesen bien registrados. Una vez hecha la depuración de las fuentes y las correcciones necesarias, se realizó vaciado de información a una matriz de datos en Excel (archivo plano) desde donde se clasifico y codifico para descubrir categorias descubrir subcategorias (tendencias), siguiendo un método de comparación constante entre los códigos. De los resultados como evidencia del proceso se espera pueda orientar la construcción de una guía clínica con que pueda ser útil en principio al CIAF y a otros centros.

\section{Resultados.}

Es importante para la comprensión del contexto de la lectura de los hallazgos aclarar que, el foco de búsqueda fue guiado por la lógica de lo que se considera intervención o tratamiento en adicciones contenido en estudios que integraron aspectos propios de lo que pudiera comprender como la psicología dinámica, entiéndase por tal aquellos trabajos que contienen desarrollos o postulados tanto freudianos como post freudianos, si retomamos a Yllá (2005) citado en (Pedrero pérez \& Et., al, 2008), este nos plantea una división para la comprensión de lo amplio en la intervención de las orientaciones psicodinámicas y en ese sentido que nos plantea que dentro de las terapias de base dinámica o analítica existen varias modalidades que apuntan al tratamiento; veamos: psicoanálisis clásico, psicoterapia dinámica o analítica (breve, focal o estándar) y psicoterapia analítica de grupo." (Pérez \& al., 2008, pág. 87), pero que no será el objetivo de este trabajo entrar a hacer tales distinciones; que bien pudiera ser entonces el objetivo de otras indagaciones. Es de anotar que las pistas de búsqueda se centraron alrededor de lo que fuera intervención o tratamiento del consumo de drogas.

Como resultado del trabajo realizado emergen varias categorías de las cuales y para efectos de esta presentación se trataran cuatro (4) de ellas con sus respectivas subcategorias o dimensiones.

\section{Categoria 1: La responsabilidad en las búsquedas del consumo.}

Tres dimensiones o subcategorías emergen en relación a las búsquedas, la primera hace alusión a un intento de resolución en el consumo; la segunda a las respuestas asociadas a momentos y en tercer lugar el consumo como una forma automedicación. En este sentido se puede plantear a partir de los textos revisados, que las búsquedas en los consumos trascienden el mero estado placer/displacer producido por la sustancia o dicho de otra manera, la estimulación del sistema de gratificación activado por el consumo y que las búsquedas se dan bajo un proceso de elección; elección de la cual el sujeto es responsable; tal aseveración se puede apreciar cuando se dice que "la toxicomanía es una elección y que de esta elección el sujeto es responsable." (Moragues Bolado, 1999). Podría decirse que el consumo hace alusión a un problema multidimensional en el que se intensifican los síntomas, sobre todo cuando el consumo se expone a estímulos que el sujeto asocia a las experiencias traumáticas pre-consumo, razón por la cual el sujeto que consume busca liberarse, siendo la droga una especie de paliativo o cura momentánea; asumiendo la 
ingesta, como condición sine qua non de relación y de evasión; en ese sentido podemos ver en el consumo un intento de salida, veamos.

\section{El consumo; un intento de salida.}

Un primer asunto a plantear y de acuerdo a lo encontrado en la pesquisa, es el hecho de que el consumo de la droga no es una elección cualquiera, pero elección al fin y al cabo de la cual el sujeto es responsable. Una elección que en algunos casos está encaminada a evitar un malestar, o dicho de otra forma evitar la división subjetiva.

"Es por tanto una elección, no una elección cualquiera ni para cualquiera, es una elección destinada a evitar el malestar, a evitar la evidencia de la división subjetiva, no es una elección que genere pérdida o percepción de la falta en ser que caracteriza a todo ser humano desde su constitución, sino más bien una elección que completa, o mantiene la ilusión de completud, sin fisuras" (Isella, 2002, pág. 43).

"La elección de la droga lejos de ser una enfermedad, es un intento de cura. Por ella el sujeto viene a suplir un defecto en la función que le constituye como tal, función paterna cuyo desfallecimiento enfrenta al toxicómano a su dificultad de relación al Otro sexo y al Otro de lo social, llevándole a buscar en la droga un sustituto o un soporte de tal función. 'Colocado', colocado en el Otro” (Arnal, 1991).

Desde otro lado se nos plantea, que las fuerzas que impulsan al consumo para la producción del placer, son en igual medida de intensidad en la búsqueda de evitar el dolor y las penas...

"La presencia de una incesante búsqueda de dicha, de la felicidad, del placer, de la satisfacción; y con esa misma fuerza la supresión del dolor y las penas. Por otra parte, podemos observar cómo esa búsqueda ha llevado al ser humano a utilizar los más variados métodos que implican la alteración de las sensaciones, de la percepción de la realidad y especialmente de lo que sentimos, para lo cual un camino (fácil) ha sido la intoxicación" (Velosa, 2009, pág. 115).

Se podría plantear que dentro de las búsquedas en el consumo está precisamente el intento de salida; una respuesta al dolor, a la división subjetiva o en general la evitación de un malestar subjetivo; búsqueda que al fin y al cabo llevan al sujeto al dolor mismo y por ello mismo nos diría Isella (2002) que en el marco de un espacio terapéutico dirijamos la intervención hacia la "transformación subjetiva", la cual produce una modificación de la dinámica psíquica, donde la abstinencia se convierte en un objetivo secundario, y que el sujeto bajo esta perspectiva puede tener otros centros ya que este habrá cambiado su posición con respecto al consumo, aunque pueda hacerlo después de manera esporádica; el beneficio radicaría en que el espacio que era ocupado por el objeto droga podrá a hora ser ocupado por la pareja, los familiares, el trabajo, el deporte en otras ocasiones, las instituciones. Teniendo en cuenta esta razón de la búsqueda en el consumo bien se pudiera disponer de ciertas estrategias para su intervención, verbigracia aprovechar los beneficios de la relación transferencial que se convierte en una herramienta fundamental ya que prohibir la droga en términos de abstinencia es simplemente mantenerla lejos, sin permitir hacer el duelo por lo que sería perderla, se plantea entonces un intento de cura pero como 
tal es fallido por cuanto queda atrapado en ella misma pues la cura como tal es aquella se inicia y puede prestar una configuración nueva a los síntomas y precipitar un nuevo estatuto subjetivo que puede ser posible por la vía de la transferencia, que sitúa al sujeto en el corazón de una nueva dialéctica.

Pero que "su efecto resulta duplicado cuando un individuo se presenta revestido de un montaje de toxicomanía que parece chocar, por su constitución misma, con el dispositivo de la cura y si, un montaje de toxicomanía no puede mantenerse intacto cuando se enfrente con el dispositivo del análisis, este se encuentra igualmente constreñido a inventar más que nunca su propio lugar" (Le Poulichet, p.148).

\section{Respuestas asociadas a momentos en el consumo.}

La pregunta sobre ¿Qué busca el sujeto en el consumo? No puede ser respondida de manera global para todos los casos, entendiendo que cada uno partiendo de su subjetividad se relacionan de maneras diferentes con su consumo; y además con el atenuante que si analizamos un solo sujeto, este estará en búsquedas diferentes dependiendo del momento del consumo por el cual este pasando. A continuación se trabajaran dos postulados los cuales se complementan en pro de la comprensión de la pregunta ¿qué busca el sujeto en el consumo?

Como ya se planteó, dependiendo del momento por el que transite el sujeto en el consumo, sus búsquedas estarán puestas en lugares diferentes. A continuación se nos plantean tres momentos en el proceso que puede llegar a conformarse como una relación adictiva con una sustancia:

Momentos según López: "Primer momento: Implica un uso "no decidido" de ella, es decir, sin intentar situarlo a nivel de una respuesta a la existencia. Segundo momento: El sujeto escoge el camino del recurrir a las sustancias como una respuesta a la falta estructural de goce y de la falta en ser del sujeto (esto con o sin problemas "objetivos" asociados al consumo) Tercer momento: El ingreso sostenido en el tiempo y con determinada intensidad provoca un cambio en el funcionamiento real del organismo, por fuera del ámbito de lo simbólico y lo imaginario, y que lleva a que se produzca la verdadera "falta relativa de control" que clínicamente habría que distinguir de algún grado de habituación posible de encontrar en el momento dos, pero que aún responde principalmente al plano subjetivo" (López, 2006, pág. 72).

Lo que López nos plantea entonces es que en un primer momento la relación con la sustancia no compromete ningún aspecto crucial en la vida psíquica del sujeto, y que por el contrario puede estar asociado a la búsqueda de placer, las relaciones de socialización en los contextos de consumo y la identificación que se produce con estos espacios... En cuanto al segundo momento, la búsqueda tiene como objetivo la ubicación subjetiva frente a la falta estructural de goce y a la falta de ser del sujeto (sintoma); ya para el tercer momento el sujeto se deslinda del inconsciente, ya que dicho síntoma ahora responde a un plano biológico.

Desde otro lugar se nos plantea que lo que se ponen en juego en el consumo de sustancias son procesos psíquicos y fisiológicos que trascienden o mutan de la búsqueda inicial del placer. En este sentido se presentan dos momentos... 
Para el primer momento en Ingelmo, se nos dice que...la droga, desde el comienzo mismo del consumo, determina, de forma casi inmediata... la desaparición del sufrimiento específico que pudiera existir previamente, provocando, al mismo tiempo, la aparición de un estado de placer, que no es mero alivio del sufrimiento previo, sino que tiene también una dimensión claramente placentera (Ingelmo, 2000, pag, 6).

Aquí el autor nos plantea que el placer producido por el consumo, muy asociado al plano de la sexualidad tiene una relación directa con las representaciones del "sufrimiento específico inicial". Además nos expone relatos donde se plantea lo profundo que puede llegar a impactar al sujeto estos estados placenteros producidos en las primeras ingestas...

Pareciera ser que se estuviera refiriendo a las experiencia que los drogadictos denominan "flash" y que, según ellos, recuerda, en parte, al orgasmo y, en parte, a la experiencia mística, y cuyas representaciones, en estos momentos iniciales del consumo, están temáticamente relacionadas con las representaciones del sufrimiento específico inicial: por ejemplo, fantasías de poder o de éxito en los sujetos con sentimientos de inferioridad y/o vergüenza (Wurmser, 1984; Lewis, 1987).

Vale recordar ahora las palabras de un consultante consumidor de bazuco cuando decía que llevaba 35 años en el consumo buscando la sensación que experimentó la primera vez y que nunca jamás lo ha vuelto a encontrar...Pareciera ser entonces que el placer inicial tiene mayor importancia, de cara a la continuidad del consumo (y, por tanto, de cara a la constitución del deseo de consumir), que el alivio del sufrimiento específico que existía previamente, aunque, pareciera también que este aspecto de alivio del sufrimiento también interviene como motor del consumo posterior. En esta línea, Rado (1933) afirma que "si la sustancia y la dosis fueron bien elegidas, el primer efecto farmacogénico placentero es por lo general el acontecimiento más impresionante de esta clase en todo el curso de la enfermedad" (Ingelmo, 2000, p. 76).

Se podría apoyar tal tesis con una referencia al opio que hace (Escohotado, 2005) quien presenta en su texto el relato autobiográfico de un inglés comedor de opio que a propósito de sus experiencias con dicha sustancia, planteaba que si sus dolores hubieran desaparecido resultaría banal a sus ojos ese efecto negativo que se había absorbido en la inmensidad de los efectos positivos que se abrían ante él, en el abismo del divino deleite que así de súbito se revelaba (...) lo que daría cuenta que ese era el secreto de la felicidad, del que los filósofos habian discutido durante tantos siglos, por fin descubierto.

En un segundo momento según Ingelmo...pareciera que el consumo se convierte ahora en el único proveedor de placer, abandonándose en él en aras de satisfacer todos los tipos de deseos desde esta conducta en donde el consumo que era usado para obtener estados de placer relacionados temáticamente con el sufrimiento con que inició el consumo, comienza a ser utilizado siempre que el sujeto desea obtener algún tipo de placer, abandonando, en aras del consumo, las actividades concretas que permiten satisfacer los diferentes tipos de deseos.

Está búsqueda activa de un estado placentero (o de incremento de un estado ya existente), y no la mera desaparición del displacer, es la que determina que algunos sujetos recaigan en el consumo de drogas en los momentos de euforia: el estado placentero existente en un 
momento determinado reactiva (memoria afectiva) las experiencias de placer, entre otras, y de manera especial, la conseguida a través del consumo de la droga” (Ingelmo, 2000, p. 7).

Se podría decir que la función del consumo para el sujeto tiene un contenido temático y especifico más profundo, lo cual hace que este busque diferentes y subjetivas formas de sentir placer, lo cual dista del placer que demanda el sistema motivacional, el cual parece responder al plano biológico.

“...la droga hace desaparecer las representaciones displacenteras, tanto conscientes como inconscientes, que pudieran existir en el consumidor, y que, obviamente, tendrán un contenido temático concreto y específico, diferente de un consumidor a otro en función del sistema motivacional que está implicado de forma predominante en la determinación del consumo" (Ingelmo, 2000, págs. 6,7).

Dos aspectos importantes para resaltar en relación a los objetivos según momentos del consumo una es que mientras para Lopez en el primer momento: hay un uso no "no decidido" de la sustancia y el consumo se hace casi sin intentar situarlo a nivel de una respuesta a la existencia, para Ingelmo, el comienzo mismo del consumo, determina, de forma casi inmediata la desaparición del sufrimiento específico y se convierte en el acontecimiento más importante en todo el curso mismo de la enfermedad, lo que llama la atención es que en la clínica con usadores de sustancias se reportan ambas situaciones por lo menos desde lo manifiesto; ya para el segundo y tercer momento las respuestas son más evidentes y se plantea en términos de una respuesta a la falta estructural de goce y en un plano más biológico anulando cualquier acto simbólico.

\section{El consumo como una forma automedicación.}

Otro emergente y muy de la mano de los dos anteriores es el uso de la droga como una forma de escape, una especie de colchón o sustituto, o "automedicación", que en algunos casos hacen referencia a aspectos emocionales o psicológicos, es el caso de un adolescente que después de unas pocas cervezas puede disfrutar de la fiesta en vez de sentirse atrapado por la ansiedad o el caso de un hombre al que la depresión le puede mejorar lo suficiente con cocaína como para permitir una interacción social, o el de una mujer que habiendo sido abusada puede tener relaciones sexuales después de medicarse con alcohol o heroína, este tipo de casos son fácilmente visualizados en la clínica con pacientes adictos a sustancias, "La hipótesis de la automedicación se confirma constantemente cuando se escuchan los relatos que hacen los pacientes de cómo responden a los estados afectivos intolerables mediante el uso de drogas." (Johnson, 1999, pág. 4). Es decir que los pacientes recurren al consumo de drogas para poder tolerar los estados afectivos intolerables para ellos.

Por su parte y lo que podría dar "sentido a la afirmación de que [...] es la adicción una forma de automedicación que tendría como fin aliviar o controlar sufrimientos emocionales intolerables Mino Annie citado en Marín (2011), mientras tanto Khantzian (1997) plantea la hipótesis de que la preferencia por una droga supone algún grado de especificidad psicofarmacológica. 
Los opiáceos por ejemplo atenúan los sentimientos de cólera o violencia, el alcohol y los depresores del SNC alivian los sentimientos de aislamiento, vacío y ansiedad y los estimulantes (anfetaminas, cocaina, etc.) mejoran la hipotonía, alivian la depresión o contrarrestan la hiperactividad y los déficits de atención." (Guerras, 2008, pág. 3); Así mismo se plantea que "...el mundo delirante podría "poblar la vida de amenazas, de sospechas, el asedio de los fantasmas de despedazamiento corporal, la intensa angustia persecutoria y el afán de calmarla, puede llevar a una intensificación del consumo de drogas. En el mismo sentido el tóxico este suele ser utilizado como un modo de automedicación, para evitar la irrupción de un goce más mortífero o en todo caso para aplacarlo, para escapar de la locura o la muerte. "El consumo también otorga un lugar de identificación que permite manejar momentáneamente el sentimiento de aniquilación” (Disanto, L citado en Ressia, 2011, pág. 202).

\section{Categoria 2: La Decisión De Buscar Ayuda.}

Lo que evidencia esta categoría es que existen perspectivas amplias de motivaciones y situaciones vitales del sujeto que lo impulsan a la búsqueda de ayuda en un espacio terapéutico y que se puede apreciar en varias subcategorías que emergen de análisis y que hacen referencia a la bondad de adecuar el tratamiento a la motivación del sujeto, tanto así como la de la inclusión de los terceros significativos en la demanda, pero también aquello que se convierte en un reto para la intervención y al mismo tiempo un serie de limitaciones para establecer la demanda.

Es de anotar que la demanda se juega entre la decisión propia del sujeto de tratarse o factores externos que lo movilizan para hacerlo. En algunos casos cuando se refiere al factor interno, como lo señala (Bousoño \& et, al., 2011) se debe a la caída de la función del tóxico del lugar de la plena satisfacción, o dicho de otra manera, la relación del sujeto con el tóxico ha entrado en fisura; sumado al planteamiento de (López, 2006) quien menciona la aparición de la angustia como motivación principal para buscar ayuda, ya que se ha perdido o se le ha escapado la respuesta en la adicción; ya en cuanto a factores externos, según (López, 2006) la influencia de "los terceros significativos" es preponderante por encima de otros, pues estos son actores protagónicos en la decisión del sujeto de tratarse, señalando que su participación en los primeros momentos del proceso facilita la adherencia y el alcance de toma de distancia con relación a los dichos iniciales del paciente sobre su adicción; veamos.

\section{Adecuando el tratamiento a la motivación del sujeto.}

Para el tratamiento es fundamental que se integren las motivaciones del sujeto y de su contexto con respecto a los objetivos terapéuticos, por tanto, será entonces necesario trabajar bajo un modelo flexible, el cual tiene como característica la capacidad de dar cabida la relación entre los objetivos del tratamiento y las motivaciones del sujeto para buscar integrarlas y adecuarlas en un solo objetivo "la propuesta se centra en encontrar la motivación del paciente, encontrar qué es lo que realmente lo moviliza "...adecuar el tratamiento a la motivación ya existente en el cliente, en lugar de adecuar la motivación a la forma de tratamiento" (López, 2009, pág. 121). 
Por tanto el objetivo es integrar una etapa que López menciona como "etapa motivacional" a la intervención misma, todo con el objetivo de poner en diálogo a la motivación del sujeto y la terapia, haciendo especial énfasis en la alianza terapéutica para construir "metas compartidas" y desde esta perspectiva...

No se busca motivar al sujeto a que acepte entrar a tratamiento, sino que se lo trata, desde el comienzo, ofreciéndole formas de tratamiento que sean aceptables para el grado y tipo de su propia motivación en ese momento, colocando énfasis en la alianza terapéutica, la actitud receptiva del terapeuta y el establecer metas compartidas (Tatarsky, 2003).

Esto cobra importancia por cuanto uno de los puntos que tiende a ser conflictivo al momento de negociar la aceptación de una forma de tratamiento, es que éste implica casi siempre la abstención completa del consumo de sustancias, lo que hace que se pierda en muchos casos, la posibilidad de generar algún vínculo que permita ir haciendo logros intermedios y/o de avanzar hacia etapas posteriores de la psicoterapia.

Con el fin de lograr dimensionar lo complejo de la instauración de la demanda de tratamiento, es necesario entender cómo llega el sujeto a terapia y el contexto que rodea la demanda, ya que estas condiciones darán matices importantes para el tratamiento y esto tiene que ver con la forma en que se logra que el miembro con el problema tome la decisión de entrar, ya que se ha visto que esto influirá en la manera en que se desarrollará el tratamiento (Loneck, Garret \& Banks, 1996) y de lo anterior se puede deducir que no sólo es relevante, lograr que el sujeto decida entrar a tratamiento, sino también la forma en que ésta, se ha producido (Landau, 2004). El pedido de tratamiento generalmente surge cuando hay un fracaso en la operación pharmakon, cuando no les garantiza la anestesia o cuando la prótesis no es la más adecuada (Djambolakdjian, 2007, 171).

En este sentido se podría plantear que al darle un lugar a la demanda del sujeto, este podría guiar y construir en la consecución de los objetivos de este “... la diversidad de estas demandas y a la particularidad de cada caso, condiciones de fundamental importancia en tanto permite orientar la dirección de la cura y las intervenciones necesarias para su implementación." (Fernández Raone, 2011, pág. 80). La solicitud de un tratamiento en estos casos constituye el primer paso de la demanda. El hablar de las peripecias con las drogas es el primer paso en una dirección transferencial, durante el cual los sujetos parecen examinar la capacidad del analista para escuchar palabras tóxicas (Djambolakdjian, 2007, 171). Por todo lo anterior se puede decir que es de gran relevancia el entender los procesos involucrados en la etapa motivacional, ya que de la forma en que se toma la decisión y los elementos subjetivos que se involucran influirán en el desarrollo del tratamiento.

\section{La inclusión de los terceros significativos; un reto para la intervención.}

Comprender la tensión que se genera entre las motivaciones internas y externas en un sujeto que consulta por una adicción se convierte en un reto para la intervención, ya que la integración de las motivaciones del sujeto y la de sus terceros significativos permitirá la orientación de los objetivos a alcanzar dentro de la terapia. 
En estos abordajes motivacionales, ya sea centrados en lo individual, en los terceros significativos o en una combinación de ambos, inevitablemente es necesario plantearse la tensión que contrapone lo que se podría llamar una motivación "propia" de parte del potencial paciente y lo que sería una motivación "espuria" producto de la influencia emocional o material de los otros: la libertad individual v/s la coerción social. Dadas las condiciones reales en que se produce el consultar en este tipo de patología, esta tensión sería parte constitutiva de la situación previa a la consulta, asi como de las primeras etapas de la intervención terapéutica. (López, 2009, pág. 122).

De lo anterior se puede decir que esta hace referencia a una tensión que es necesario entender más que evitar en nombre de ciertas posturas que defienden la libertad de elegir a toda costa por parte del paciente, o la posición contraria en la cual la imposición de un patrón de conducta supuestamente adaptativo a cualquier costo sobre el paciente. El tema es un poco complicado en tanto que si bien la efectividad de los tratamientos parece requerir una apuesta por parte del sujeto, también es cierto que dada la influencia y potencia de los deseos de consumo asociados al desarrollo de una adicción a sustancias químicas, normalmente es problemático que el paciente con el problema se involucre en una decisión de tratarse sin que otros significativos estén influyendo sobre él.

Con respecto a lo anterior, se pueden evidenciar dos aspectos recurrentes en las intervenciones de sujetos con problemas de consumo: Primero que no consultan, y como segundo que cuando decide tratarse, participa con una motivación impuesta o movilizada por terceros; sólo un porcentaje minoritario de la población con problemas de abuso o dependencia consulta en algún momento de su vida (Cunningham \& Breslin, 2004). Es así como estudios realizados en EEUU y Canadá calculan que, cada año, entre el 90\% y el 95\% de las personas con dependencia al alcohol y/o drogas no entra a tratamiento o a grupos de autoayuda (Landau, y otros, 2004 et al., 2004). Por otra parte, y como una dificultad adicional, en un porcentaje importante del grupo que consulta la motivación a consultar está dada por terceros" (López, 2009, pág. 120).

Por los aspectos tratados en este apartado se evidencia una propuesta; cual es la de reconocer y vincular a la solicitud de ayuda terapéutica las motivaciones e influencias de los terceros significativos para con el proceso. Algunos textos plantean la existencia de estudios empíricos que sustentan la participación de los terceros significativos dentro de los procesos terapéuticos; como primero para que los sujetos tomen la decisión de entrar a proceso terapéutico y como segundo para el desarrollo en general de este proceso.

La incorporación de "terceros en el proceso de consulta ha sido apoyada por estudios empíricos que muestran que el compromiso de la familia y de los amigos en el proceso de tratamiento de personas con problemas de adicción adquiere una gran importancia (Copello, Williamson,, Oxford, \& Day, 2006). Así, por ejemplo, se ha visto que pacientes que viven en pareja o con amigos y/o familiares tienen tres veces más probabilidades de entrar a tratamiento que los que viven en otro tipo de entorno ((Lloyd, y otros, 2005), por lo que se puede plantear que es importante ser conscientes que los terceros significativos pueden jugar un rol preponderante tanto en la decisión de entrar a tratamiento como en el desarrollo del proceso de tratamiento. La entrada a un proceso de intervenciones terapéuticas dirigidas al problema del consumo de sustancias como una 'decisión' por parte 
del sujeto es de vital importancia, y ha sido uno de los aportes principales de los enfoques motivacionales, sin embargo...

La inclusión de terceros, por su parte, permitirá que el sujeto fije de forma mucho más rápida una de las posiciones ya existentes en él, facilitando la decisión de entrar a un tratamiento. De esta manera, no existiría una oposición entre darle una cabida terapéutica a la demanda de los terceros significativos y el mantenimiento de una libertad de elección del sujeto con el problema que pueda facilitar un hacerse responsable y un involucrarse subjetivamente en el tratamiento que se inicia (López, 2006, pág. 126).

A partir de lo revisado en este estudio se puede decir que este proceso puede incluir la demanda de terceros significativos sin que esto afecte la dimensión de 'decisión' en juego, en la medida que terapéuticamente se le da un espacio al desarrollo de esta decisión sin perder de vista que el pedido se hace generalmente a través de un familiar que sirve de intermediario en la búsqueda de tratamiento y que la "demanda precisa ser "entre" las fuerzas de uno y de otro para poder competir con el tóxico" (Djambolakdjian, 2007, p.191).

\section{El reto para la intervención y las limitaciones para establecer la demanda.}

Cuando se plantea el establecimiento de la demanda con relación a los terceros es importante señalar que, cuando estos no son significativos a diferencia de como lo indica la categoría anterior, esta relación puede generar formaciones reactivas que hacen que dicha posibilidad se aleje de tales aspiraciones, es decir de un tratamiento, constituyéndose como un limitante digno de ser tenido en cuenta para los clínicos, y es en ese sentido cuando plantea que...

En la mayoria de los casos en los que la consulta es efectuada por los padres o por orden judicial penal o del Tribunal de Familia, son diversas las modalidades que presenta la respuesta del sujeto frente a la objetivación que recibe por la descripción o las sanciones de los otros. En general, responde con indiferencia y desinterés, o reafirmando la necesidad del uso de la droga como estilo de vida compartido por su grupo social. No se presenta una dimensión conflictiva, excepto con la autoridad que cuestiona su proceder" (Fernández Raone, 2011, pág. 81).

Lo anterior precisamente por cuanto quien impone no ha creado un vínculo que haga que este sea significativo, y por lo tanto no vehiculice alguna demanda; es más, el paciente puede incluso asistir y visitar profesionales e instituciones e incluso solicitar ayuda pero en la práctica seguirá siendo la demanda del tercero que para acabar de ajustar tampoco viene siendo significativo.

Otro asunto que se encuentra del lado de la no demanda de tratamiento tiene que ver con la falta de motivación y en este sentido se puede decir que...

"Ésta situación de falta de motivación de la persona que tiene el problema constituye un desafio a ser resuelto tanto en relación a la entrada a procesos terapéuticos como en la adherencia a ellos, más allá del tipo de tratamiento que se trate" (López, 2009, pág. 1). 
Siguiendo a Moragues Bolado (1999) se puede decir entonces que una de las mayores dificultades para iniciar un tratamiento sea, precisamente, la certeza con la que el toxicómano se arraiga en esta posición en la que a través de la droga puede explicarlo todo; le corresponderá al clínico tratar de descubrir y comprender qué tipo de respuestas busca o evade este en su consumo; por lo demás, dicho pedido casi nunca es formulado de manera directa. "Generalmente es necesario que alguien lo descifre a partir del comportamiento o atraer las miradas hacia sí a través del cuerpo casi mutilado (Djambolakdjian, 2007, 171).

Se puede decir y plantear sin temor alguno que el gran problema y el reto día a día de los teóricos y de los centros que intervienen con estas problemáticas, reclama nuevas comprensiones que posibiliten intervenciones creativas, y es aquí donde vuelve y cobran sentido los terceros que logran ser significativos, sobre todo cuando no hay una demanda propia y que estos pudieran convertirse en un paso importante hacia la adherencia a los tratamientos.

\section{Categoria 3: Hacia Una Evaluación Comprensiva Del Fenomeno.}

La intervención del fenómeno deberá apuntalarse en una visión comprensiva del fenómeno; a ello podría llegarse mediante un proceso de evaluación, el cual y siguiendo a (Kirchner, Torres, \& Forns, 1998), quienes nos lo plantean como un corpus de conocimientos teóricos y prácticos imbricados con la psicología general, pero que para nuestros efectos la entenderíamos en lo particular; esto es desde los dispositivos propios de la psicología dinámica con lo cual se persigue describir, clasificar en alguna medida predecir y modificar, el comportamiento asociado al consumo de sustancias, utilizando para ello instrumentos y técnicas que le son propios..., para el estudio en cuestión y de los textos revisados se seleccionaron varias de las dimensiones que emergen y están relacionados con el proceso de evaluación desde la perspectiva dinámica de la psicología y que sugieren ser tenidos en cuenta para pensar procesos de y son los que se encuentran relacionados con la evaluación de cambio: resolución o mutación; el control no es el objetivo central; el reconocimiento de la subjetividad en el diagnóstico y sobre una atención diferenciada.

\section{Evaluación de cambio: resolución o mutación.}

Apropósito de los objetivos propuestos, en la tradición de los dispositivos de intervención desde posturas objetivistas, es valorado aquel que apunta a la abstinencia del objeto droga y con frecuencia se encuentra en la clínica que se suspende la ingesta de una sustancia y aparece dependencia a otra o un otro comportamiento adictivo; con lo cual vale la pregunta de si la abstinencia es suficiente cuando no es que lo que esté sucediendo sea una mutación a otro objeto y allí. Los terapeutas necesitan estar alerta a la tendencia a cambiar de una adicción a otra, por ejemplo, si un paciente adicto deja las benzodiacepinas pero empieza jugar a las maquinitas desaforadamente y su situación en sus diferentes áreas se ven afectadas ¿deberá considerar esto como un éxito o como alguien cuya adicción ha cambiado a un sustituto que suele tenerse menos en cuenta?, para muchas familias y para muchos pacientes la sustitución de una adicción por otra puede ser considerada un éxito terapéutico relativo y se dice por ejemplo que...

Bill Wilson, el fundador de "Alcohólicos Anónimos", murió debido a su adicción a la nicotina pero muchos años productivos después de que estuviera sobrio del alcohol. En otros 
casos, la sustitución necesita ser tenida en cuenta como un signo de falta de capacidad para lograr una recuperación estable. Los estudios de mantenimiento con metadona, por ejemplo, tienden a usar la abstinencia a los opiáceos como una medida del resultado, a pesar que muchos pacientes continúan usando el alcohol, la cocaina o las benzodiacepinas de forma adictiva y autodestructiva (Mills y Gold citados en Johnson, 1999, pág. 18).

Respecto a la evaluación del cambio Johnson (1999) plantea la complejidad de tal situación y para tal efecto pone un ejemplo en el cual deja dicho que...

Una mujer adicta a la comida, con atracones/purgas puede cambiar a la dependencia de la cocaina porque le permite mantener su peso bajo, y puede posteriormente simplemente preocuparse a ratos de cómo obtener, usar y recuperarse de la cocaina. Un hombre que tiene problemas porque bebe puede cambiar al juego compulsivo porque, por lo menos durante un tiempo, puede desarrollar mejor su conducta compulsiva. Cuando conseguir la heroina se vuelve demasiado trabajo, a medida que las personas envejecen pueden cambiar hacia la dependencia del alcohol" (Johnson, 1999, pág. 7).

Para no ir muy lejos basta con remitirnos al primer tratamiento freudiano de la toxicomanía, cuando este propuso un tratamiento de sustitución de la heroína por la cocaína, muy similar al practicado hoy en día en los establecimientos psiquiátricos, donde se proporcionan medicamentos como la metadona para alivianar el síndrome de abstinencia en la cura de desintoxicación de las llamadas "drogas duras", heroína y cocaína (Renjifo, 2007). Para la mayoria de las personas y sobre todo aquellas que representan al sector salud y se puede decir que a nivel Global y local (glocal) la abstinencia es el indicador máximo pero al mismo tiempo la razón del desánimo de tantos esfuerzos para para ver que no es un porcentaje muy elevado de los que logran mantenerse abstemios, para otros y muchos que hacen parte del gremio de los profesionales la pregunta es de hasta dónde la suspensión de un objeto y el reemplazo por otro sí es recuperación, aunque la verdad muchos se conforman con esto; pero aun así también algún sector cree que el único énfasis no debe ponerse solo sobre el objeto droga.

En este sentido y tal vez escuchando un poco más sobre el papel de las sustancias en las emociones y sentimientos, se pudiera contribuir con algo más en la durabilidad de los cambios; esto es que si se prestan oídos al papel que desempeñan las drogas en relación a los sentimientos y comportamientos problemáticos, y de cómo se pueden resolver los problemas sin recurrir a las drogas, se puedan promover cambios duraderos en el estilo de vida (Woody, McLellan, Luborsky y O’Brien, (1995) citados en" (Pérez \& al., 2008, Pág. 87). En todo caso y como ideal no deberá tratarse de cambiar un tipo de adicción por otro; o tal vez sí pero como un paso intermedio hacia un proceso de transformación global.

\section{El control no es el objetivo central.}

Es común en la clínica como en la cotidianidad, no menos en los trabajos revisados, que un énfasis es el de la pérdida del control en relación consumo de sustancias; así las cosas, "La adicción la podríamos caracterizar, en primer lugar, por lo que se podría llamar una "falta de control relativa" sobre la conducta del consumo de sustancias” (López, 2006, pág. 67). 
Sin embargo la invitación de las orientaciones psicodinámicas en relación a los tratamientos en las adicciones, no es el del énfasis sobre la falta de control de las sustancias, sino el de la identificación de aquello que el sujeto puede controlar y lo que no en su psiquismo; más bien se podría plantear que en la práctica psicoanalítica, el valor terapéutico, en el sentido noble de esa palabra...

Es que el sujeto pueda dignamente convivir con las tres condiciones que le han sido dadas que son: ser sexual, ser parlante y ser mortal, condiciones... Estas tres cosas hacen plantearse el problema del obstáculo, de lo que no anda en la vida... con lo que en la vida no se puede manejar. Por eso a veces los individuos hablan de la falta de control, pero la paradoja es que el psicoanálisis no busca cómo controlarlas, sino como poder diferenciar lo que se puede controlar y lo que no y a qué distancia puede uno ponerse en todo caso respecto a aquellas cosas que no controla Sergio (2001) citado en (Isella, 2002, pág. 62)

La sustancia, como objeto, comienza a ocupar un sitial privilegiado en el psiquismo, lo que lleva a que la vida, a nivel conductual, cognitivo, emocional, y relacional, comience a girar en torno de la obtención de la sustancia, o la expectativa del placer asociado, o la generación de las condiciones para su consumo por lo que se podría decir entonces que el diagnóstico comprensivo desde las orientaciones psicodinámicas se concentran en los aspectos que el sujeto puede y no puede controlar dentro de tres condiciones que se le han otorgado, pero por otro reclama tener en cuenta el impacto del consumo en el sujeto.

\section{Reconociendo la subjetividad en el diagnóstico.}

Donghi propone que el objetivo del diagnóstico en el psicoanálisis tiene como propósito no solo rotular el comportamiento sino además, que haya un reconocimiento de la importancia en el efecto causal o etiológico por parte de la sustancia. Aunque de más difícil aplicación, la ventaja mayor del diagnóstico psicoanalítico es que permite ubicar la participación inconsciente del sujeto en la creación y sostén de su sintoma. Es esa toma de posición a su vez lo que permite orientar la intervención del analista y en ese sentido que se puede agregar que...

El diagnóstico psicoanalítico revela la implicación causal del sujeto en la producción del sintoma y constituye ya un principio de separación y responsabilizarian al sujeto, en la medida en que lo extrae de su implicación alienada en el síntoma. Esta participación del sujeto en la etiología del sintoma conlleva per se efectos terapéuticos y un pasaje del pánico colectivo y las formas extraviadas de la angustia, a una angustia más personalizada, en la que el adulto tiene alguna chance de responder con las referencias estructurales y recursos de que dispone (Donghi, 2006, pág. 56).

Este proceso precisa que el terapeuta explore las representaciones subjetivas del paciente, entendiendo desde el marco conceptual del sujeto su forma de ver el mundo, para dar sentido a la formación del síntoma como expresión inconsciente para que este tome posición frente a su situación.

"El proceso diagnóstico psicoanalitico consiste en el trabajo por el que el analista se ubica en el campo transferencial del paciente, para hacer posible desde alli una manifestación 
más nitida del sintoma en tanto expresión de un saber inconsciente que concierne y divide al sujeto que lo padece (Lacan, 1965). El resultado del proceso no es solamente una etiqueta o código diagnóstico, sino una puesta en forma del sintoma en un vínculo transferencial, que supone al menos una experiencia del inconsciente” (Donghi, 2006, pág. 56).

Siguiendo a (Renjifo, 2007), este plantea que la adicción no puede ser considerada como una entidad aislada de la configuración subjetiva de un individuo y que la apuesta de intervención deberá ser orientada hacia la interrogación sobre el estatuto del consumo y sus efectos en cada sujeto cuando las posibilidades se dan, lo que sugiere que hay algo del orden del deseo en el sujeto que introduce la pregunta, abriendo al mismo tiempo la posibilidad de restablecer el lugar del objeto de la necesidad (el tóxico), por la falta en que se disfraza el objeto que causa el deseo.

Es por esto y que en caso de continuar con las entrevistas, estas se orientan a ubicar cuál es la función que cumple el tóxico para el consultante. "Ese elemento diagnóstico es esencial para determinar la orientación del tratamiento con ese paciente." (Bousoño \& al., 2011, pág. 29), todo ello por cuanto la función para pacientes y clínicos- toxicómanos y médicos- estos se ha organizado como "saberes saberes en torno de la figura del tóxico, anulando la perspectiva de una posición de los sujetos” (Le Poulichet, 2005, p, 35-36).

\section{Sobre una atención diferenciada.}

Los hallazgos muestran que autores recomiendan que se debe realizar énfasis en el diagnóstico estructural, ya que este permitirá comprender la función y diferencias en el impacto psíquico de la sustancia, con el fin de orientar el tratamiento: En múltiples ocasiones se referencia que algunos de los síntomas clínicos pueden ser ocasionados por el efecto de la sustancia o como mera expresión de la estructura, por tanto es necesario realizar una distinción entre la etiología de los síntomas, es de anotar que en la clínica con pacientes adictos se presentan dificultades diagnósticas respecto de la estructura que subyace a la adicción; así algunos manifiestan un funcionamiento psicótico aunque no se presenta una psicosis clínica propiamente dicha (...) y también es cierto que "surgen una serie de fenómenos psicopatológicos que llevan a interrogar si los mismos son consecuencia del consumo, o si bien se corresponden con su estructura de personalidad de base" (Ressia, 2011, pág. 202).

Otro autor que apoya esta postura es Guerras, quien platea que el diagnóstico diferencial le permitirá al terapeuta guiar la intervención según las lógicas estructurales cuando deja claro que...

Es de vital importancia el diagnóstico de estos pacientes a nivel estructural (neuróticos, limites o psicóticos), ya que el trabajo que se pueda realizar con ellos va a depender en gran medida de su estructura de personalidad, a mayor desestructuración del paciente mayor estructuración del tratamiento (Guerras, 2008, pág. 16).

...Y se podría agregar que no solo una evaluación-intervención diferencial según sea la estructura sino también del tipo de sustancia usada que podría variar seguramente también asociadas al tipo de estructura; a modo de sintesis se podría decir que la relevancia de realizar un diagnóstico estructural no está puesta en el etiquetamiento del sujeto, sino del sentido de 
orientación que esta le posibilita a la intervención, en este sentido se puede decir que el rótulo si bien no es lo más importante, si da un orientación; pues "no es lo mismo intervenir a una persona con una neurosis que con psicosis, habrá que "determinar cuál es la estructura clínica para desarrollar la técnica” Walter Cuartas citado por (Marín, 2011, pág. 11).

\section{Categoria 4: Sobre la intervención.}

Orientaciones psicodinámicas han sido objeto de críticas bajo el argumento de la ausencia de intervenciones basadas en evidencia científica; ya que en el ámbito de la psicología se le da favoritismo a las perspectivas de intervención que se centran en la modificación de comportamientos; aun así vale la pena volver la atención sobre los presupuestos teóricos y objetivos terapéuticos, así como de las particularidades de cada sujeto a la hora de implementar los dispositivos de intervención, los límites y alcances de la misma; a continuación varias de las categorias que emergen del rastreo realizado: sobre la Orientación de la acción terapéutica, los Alcances y límites del tratamiento psicodinámico, los objetivos del tratamiento y algo sobre técnicas y métodos.

\section{Orientando la acción terapéutica.}

Según el comité de expertos de la Organización Mundial de la Salud (OMS), el tratamiento se define como el "proceso que comienza cuando los usuarios de sustancias psicoactivas entran en contacto con un proveedor de servicios de salud... y puede continuar a través de una sucesión de intervenciones concretas hasta que se alcanza el nivel de salud". (Naciones Unidas contra la Droga y el Delito, 2003)" (Sarria F., 2012, pág. 16); siguiendo a Sarria (2012) el tratamiento puede ser definido, como una o más intervenciones estructuradas para tratar los problemas de salud y de otra índole causados por el abuso de drogas y aumentar u optimizar el desempeño personal y social. El tratamiento de las adicciones desde las orientaciones dinámicas no debería centrarse en el cese del consumo lo que no significa que...

El sujeto deje de consumir como condición para iniciar el tratamiento o continuarlo, sino que el consumo pase al fondo de la relación terapéutica, para que pueda comenzar a hablar de otras cosas que le pasan, que le preocupan o le generan algún tipo de sufrimiento, que la droga ha venido momentáneamente a calmar; es decir la finalidad es que cada sujeto tome conciencia de su particular posición subjetiva (Isella, 2002, págs. 43,44).

Cuando se habla de las intervenciones desde la clínica con perspectivas psicodinámicas se puede plantear que...

...fundamentalmente se hace una escucha activa de lo que está sucediendo en ese momento puntual de la vida del sujeto y de tratar de entender el papel que juega la sustancia en su historia de vida. La intervención desde la clinica individual parte siempre de la demanda del individuo; desde el deseo, y en ese sentido si una persona solicita una consulta, es porque está haciendo una propuesta de recuperación para resolver el porqué de los interrogantes en su vida (Marín, 2011, pág. 3). 
La la intervención deberá enfocarse en otros aspectos como que el tratamiento tiene que ver con la clínica del caso por caso, basada en la escucha analítica, para que la droga caiga precisamente de ese lugar de solución y de totalidad para que pueda dar lugar a que aparezca algo del orden de lo sintomático, algo que comience a cuestionar, que manifieste otro malestar distinto al relacionado con el consumo.

\section{Alcances y limites del tratamiento psicodinámico.}

Una de los aspectos que podría dar cuenta de lo pertinente del tratamiento desde las orientaciones psicodinámicas, sería cuando un sujeto se le realizan diferentes tipos intervenciones basadas en el síntoma, pero estás no logran ayudar al sujeto en relación con su padecimiento y además de percibir el malestar en su paciente pero que este no es generado netamente por lo fisiológico, sino que la génesis ha de buscarse en el campo psíquico, para lo cual será necesario entonces realizar una intervención desde un modelo comprensivo del consumo y la relación que esta tiene con la historia de vida del sujeto.

Parafraseando a Sarria, F. (2012) el enfoque psicoanalítico no sólo permite una intervención desde el plano clínico habitual, sino también desde lo social (topología del sujeto), además este autor nos plantea que el modelo psicoanalítico no centra su atención en el tratamiento del objeto sino más bien sobre el discurso del sujeto y sus relaciones con los demás:

...El punto de partida de una intervención que no está basada en la premisa de una abstinencia sino en la demanda de atención de un sujeto regido por las leyes del lenguaje del cual surge; da paso a la lectura del cómo, en su discurso, el consumo está asociado a sus relaciones sociales, su familia, sus amigos (Sarria F., 2012, pág. 20).

"E1 Psicoanálisis, en tanto experiencia dialéctica, se propone como una herramienta eficaz para el abordaje de la clínica con toxicomanías." (Marín, 2011, pág. 28); además se sugieren que la intervención desde el psicoanálisis convencional es útil para sujetos en "estados avanzados de recuperación y para tratar rasgos de personalidad anómalos asociados o previos a la adicción.” (Becoña Iglesias \& Cortés Tomás, 2008, págs. 87,88); en estos casos se puede realizar un tratamiento desde el enfoque de la psicología dinámica con "mayor silencio del terapeuta, esta terapia puede ser muy efectiva, porque posibilita devolverle al otro el lugar de la pregunta y el lugar de la elaboración, ya que el paciente toma una posición frente a su existencia al hacer insight." (Marín, 2011, pág. 10).

Así las cosas también se tendría que tener en cuenta que la "toxicomanía es una entidad clínica que no se constituye como un clásico síntoma freudiano. Es decir como la expresión metafórica de una satisfacción que fue reprimida y relegada a lo inconsciente. En este caso el sujeto ha encontrado un objeto exclusivo y excluyente que lo colma o lo colmaba de satisfacción. Una satisfacción inmediata que tiene un lugar de certeza para él" (Bousoño \& al., 2011, pág. 28).

Ahora y dentro de algunos de los obstáculos para atender a estos pacientes, conlleva a tener presente el contexto del paciente por cuanto en muchos casos se dificulta el tratamiento analítico; con estos pacientes un aspecto a atender consiste en ver cómo responder ante situaciones acuciantes (falta de dinero, problemas de vivienda, de salud, legales) ante las 
cuales se hace un pedido de respuesta instantánea, en términos de una solución inmediata y efectiva, para que se les den soluciones en términos de poder operar cambios concretos de su realidad cotidiana, lo que se acerca más a intervenciones de tipo social y desvirtúan la propuesta de una psicoterapia psicoanalitica. Se trata de situaciones que llevan al terapeuta a debatirse en cómo dar una respuesta que ayude al paciente en términos emocionales y que a su vez favorezca que el tratamiento continúe

Estas dificultades constituyen presiones constantes sobre el trabajo de análisis, todo lo cual potencia las dificultades para pensar durante la sesión. Debido a esta presión, en muchos casos, el terapeuta puede verse tentado de derivar el paciente por no poder proseguir con el tratamiento debido al creciente sentimiento de impotencia que lo embarga. (Ressia, 2011, pág. 203).

Por último, y a propósito de una intervención de corte psicoanalitico de tipo ambulatorio, puede complicar el asunto por cuanto aumentan las posibilidades de que los pacientes falten a las sesiones de tratamiento, debido a la hostilidad o al uso de drogas, sin embargo, los "pacientes que vienen y trabajan, a pesar de su relación con sustancias adictivas o conductas adictivas, pueden ser ayudados" (Johnson, 1999, pág. 18).

\section{Sobre los objetivos del tratamiento.}

Acerca de los objetivos de la intervención, se percibe una notable diferencia con tratamientos que privilegian la comprensión biológica o comportamental, donde el enfoque del problema con las adicciones es el objeto droga; y donde la resolución se define por la separación de sujeto-droga. Un ejemplo para ilustrar el anterior planteamiento, es el tratamiento ofrecido en los centros de Alcohólicos o Narcóticos Anónimos que implica una renuncia total al consumo o a la continuación con el objeto "proporcionada por la conducta adictiva; a adoptar mediante un "salto de fe" la creencia de que existen objetos humanos confiables; a cambiar el superyó, extendiendo el trabajo de recuperación al ideal del yo o a valores sociales internalizados" (Johnson, 1999, págs. 8-9).

El objetivo o meta terapéutica que deberá primar en el trabajo con personas que consumen drogas psicoactivas será la de dar "dar lugar al sujeto, con el fin de que pueda desplegar la trama significante que lo determina, acompañándolo a reconocerla, a reconocer las encrucijadas que se le plantearon y donde el tóxico jugó su partida. Como lo propone (Bousoño \& al., 2011, Pág. 28), esta sólo puede darse mediante la transformación subjetiva, la que se podría definir como el proceso que va "modificando la dinámica psíquica del sujeto, de manera que la abstinencia de consumo, en estos casos, pase en principio a un segundo plano, y que finalmente si surge lo haga como resultado de tales cambios" (Isella, 2002, págs. 57,58$)$.

Para el cumplimiento de este objetivo como ya se dijo anteriormente no se tiene como requisito la abstinencia, sino un trabajo arduo para que el paciente sea capaz de reconocer el malestar que subyace a esto. Por eso cuando un paciente lleva al espacio terapéutico la "certeza" de ser un adicto de... "tendríamos que cuestionarnos y cuestionarlo a él o a ella sobre su discurso. Seguramente es una reproducción del imaginario social que ha construido dicha denominación para todo aquel que consume drogas ilícitas.” (Guerras, 2008, pág. 13). 
El objetivo deberá por lo demás centrarse sobre capacidades y recursos psicológicos positivos

... Dependiendo de la persona y de las circunstancias, esto podría incluir la capacidad de tener relaciones más satisfactorias, hacer un uso más eficaz de los propios talentos y habilidades, mantener un sentido de la autoestima realista, tolerar una amplia gama de afectos, tener experiencias sexuales más satisfactorias, entenderse a sí mismo o a los otros de manera más matizada y sofisticada y afrontar los retos de la vida con una mayor libertad y flexibilidad (Olivera, 2011, pág. 173).

El objetivo terapéutico deberá entonces girar en torno a la reivindicación del sujeto que no tiene como premisa central la abstinencia del objeto, sino de un proceso que puede llevar a que el paciente sea capaz de reconocer el malestar que subyace y pueda hacer algo con eso. No deberá echarse en saco roto para el tratamiento desde orientaciones psicodinámicas la labor terapéutica orientada hacia "la reinserción y readaptación temprana del sujeto adicto a su medio, en contraposición a la segregación forzada." (Fanton, 2011, pág. 75). Retomado a Héctor Juan Fiorini cuando plantea el tema de la activación de funciones yoicas por ejemplo, para él tratamiento se representan en doble frente, en la cual se tienen presentes las partes sanas del paciente y por ende no sólo se hace referencia a los aspectos infantiles, regresivos o patológicos, sino también los aspectos sanos o área del yo libre de conflictos (Fiorini, 1973, 1987).

\section{Conlusiones.}

Respecto a las sustancias y sus búsquedas se puede concluir que

El consumo de la droga es una elección que hace el sujeto, elección de la que es a su vez responsable, dicha elección en algunos casos está encaminada a evitar un malestar, o dicho de otra forma evitar la división subjetiva; los objetivos en las búsquedas son diferentes según lo momentos; en un primer momento parece que las búsquedas no compromete ningún aspecto crucial en la vida psíquica del sujeto, mientras que en la segunda y la tercera se compromete seriamente la vida psíquica del sujeto.

Es evidente el placer producido por el consumo, frecuentemente asociado a la vida sexual; la ingesta genera un impacto placentero producidos sobre todo con las primeras ingestas de la sustancia; el consumo de sustancias de por sí no es la condición única para que en el sujeto se instaure una adicción y que son necesarias también condiciones del sujeto y de su contexto para que a través del tiempo esta se convierta en tal situación. Más precisamente, la diferencia entre consumo y adicción es cuando se fija una posición subjetiva.

Según algunos autores entre ellos Marin (2011), el consumo en ciertos casos se convierte en una forma de automedicación que tendría como fin aliviar o controlar sufrimientos emocionales intolerables. El ingreso al tratamiento es fundamental que se reconozcan y se potencien las motivaciones del sujeto y se articulen a las de su contexto y para ello parece que es necesario trabajar bajo principios de un modelo flexible; esto es adecuar el tratamiento a la motivación ya existente 
Respecto a la dificultad en muchos casos para el establecimiento de una demanda propia se hace necesario incorporar al proceso las demandas de los terceros; pero no de cualquier tercero es un tercero significativo; de lo contrario se lograría un efecto adverso.

Con Donghi se plantean entre otros, atender a los objetivos de que se dispone para la intervención y aunque acentúa sobre el psicoanálisis clásico valdría también para otras perspectivas de corte psicodinámico pero que en ambos casos, sus propósitos no centran únicamente está el del rotulamiento.

También se puede decir que el tratamiento de las adicciones desde las orientaciones dinámicas no deben de centrarse solamente en el cese del consumo; este deberá llegar por añadidura y no como el foco sobre el cual intervenir, por el contrario debe enfocarse en otros aspectos: este se basará en una escucha analítica; para que la droga caiga de ese lugar de solución y de totalidad que abarca y tapa toda dimensión subjetiva; para que se pueda caer del lugar ha de aparecer algo del orden de lo sintomático, algo que comience a cuestionar, que manifieste otro malestar distinto al relacionado con el consumo.

Existen trabajos con evidencias empíricas que sustentan la efectividad de la terapia dinámica en adicciones pero aun así, siguen siendo en cantidad menor en número en comparación con los modelos comportamentales. La terapia dinámica con respecto a las otras orientaciones ofrece un valor agregado, ya que los beneficios alcanzados en terapia lleven a que los casos mejoren y en algunos casos se sostengan con el pasar del tiempo.

Siguiendo la lógica de los planteamientos anteriores que al final del proceso conviene decir que trabajos en esta dirección y más aun con perspectivas psicodinámicas como o la que se exponen en este trabajo deberán seguir su curso por un lado es importante para calcular efectividad se deberá atender a sus presupuestos conceptuales y a los objetivos y alcances de los mismos; vemos pues que el acento y la condición de entrada no es la abstinencia y que más bien esta vaya cayendo a partir de ciertas transformaciones y su unidad de medida no deberá ser más las mismas con las cuales se miden las perspectivas hegemónicas que se autodenominan como las únicas efectivas; pues como lo plantea (Olivera, 2011); importante tener en cuenta que varios estudios dejan entrever que los efectos de esta lógicas pueden mantenerse en en el tiempo, pero también es cierto que falta mayor evidencia empírica; el hecho de que hayan pocos estudios no significan que no es razón suficiente para pensar que no genere transformaciones y transformaciones podriamos decir desde adentro; en tanto se rescata para el sujeto la palabra; lo que se propone al final de cuentas es que se pueda rescatar perspectivas comprensivas, por tanto tendrian que pensarse unos criterios de efectividad propios ajustados a sus objetivos; pareciera que se le evalúan con indicadores externos a su lógica teórica.

De otro lado y para trabajos posteriores se deberá hacer distinción clara para efectos de intervención sobre el tipo de abordaje como lo planteara (Braier , 1980) hacer la distinción entre psicoterapia Breve de orientación psicoanalítica o psicoanálisis propiamente dicho, o técnicas de reconstrucción de la estructura. 


\section{Referencias Bibliográficas.}

Arnal, J. M. (1991). Análisis de la demanda de(1) toxicómano. Ponencia presentada en la IV Jornada de Formación de la Plataforma Internacional para una Clínica del Toxicómano, en Toledo. (1991).

Becoña Iglesias, E., \& Cortés Tomás, M. (2008). Guía Clínica de Intervención Psicológica en Adicciones. Guías Clínicas Socidrogalcohol Basadas En La Evidencia Científica. Barcelona: SOCIDROGAALCOHOL Sociedad Cientifica Española de Estudios sobre el Alcohol, el Alcoholismo y otras Toxicomanías.

Bousoño, N., \& al., E. (2011). Una experiencia en el Tratamiento de las Toxicomanias. Memorias del III Congreso Internacional de Investigación y Práctica Profesional en Psicología (págs. 26-30). Buenos Aires.: Ediciones de la Facultad de Psicología - Universidad de Buenos Aires.

Braier , E. A. (1980). Psicoterapia Breve de Orientación Psicoanalitica. Buenos Aires : Ediciones Nueva Visión.

CCI-FIUC, Centro Coordinador de Investigaciones de la <federación Internacional de Universidades Católicas. (2008). DROGAS EN AMÉRICA LATINA. Estado del arte en estudios de toxicomanía en Argentina, Brasil, Colombia, Chile y Ecuador. Santiago: Ediciones UCSH.

Chavarriaga Montoya, A. (1996). Aportes Teóricos para una Comprensión del Fenómeno de la Drogadicción a partir de los conceptos de impulsión y rasgo de Perversión. Medellín.

Copello , A., Williamson, E., \& Day, E. (2006). Implementing and evaluating Social Behaviour and Network Therapy in drug treatment practice in the UK: A feasibility study. Addictive Behaviors, 221-223.

Cunningham , J., \& Breslin, F. B. (2004). Only one in three people with alcohol abuse or dependence ever seek treatment. Addictive Behaviors(29), 221-223.

Djambolakdjian, S. (2007). Clínica psicoanalitica de la toxicomanía. Desde el jardín de Freud, 161-174 Ficha No. 23.

Donghi, A. (2006). Aportes del Proceso Diagnóstico Psicoanalítico al Tratamiento de las Adicciones en Ámbitos Hospitalarios. Scielo, XIV, 55-58.

Escohotado, A. (2005). Aprendiendo de las drogas: usos abusos, prejuicios y desafios. barcelona: Anagrama.

Fanton, M. (2011). La Violencia Explícita y Encubierta en la Clínica de las Adicciones. Memorias del III Congreso Internacional de Investigación y Práctica Profesional en Psicología. (págs. 75-78). Buenos Aires: Ediciones de la Facultad de Psicología - Universidad de Buenos Aires. 
Fernández Raone, M. (2011). Adolescencia y Consumo de Sustancias: El problema de la Demanda Terapéutica. Memorias del III Congreso Internacional de Investigación y Práctica Profesional en Psicología (págs. 80-83.). Buenos Aires.: Ediciones de la Facultad de Psicología - Universidad de Buenos Aires.

Fiorini, H. J. (1973). Funciones Yoicas en el proceso terapeutico . En H. J. Fiorini, Aportes para una teoría de la técnica. Buenos Aires: Nueva Visión .

Freud, S. (1932). 34a Conferencia. Esclarecimientos, aplicaciones, orientaciones. En Obras Completas (2 ${ }^{a}$ Ed.), Tomo XXII. Buenos Aires: Amorrortu ,.

Freud., S. (1913). Sobre la iniciación del tratamiento. En Obras Completas (2 ${ }^{a}$ Ed.), Tomo XII. Buenos Aires:. Buenos Aires: Amorrortu Editores.

Galeano M, M. E. (2008). Diseño de proyectos de Investigación Culitativa. Medellón, Colombia: Universidad Eafit.

Guerras, J. G. (2008). Psicoanalisis y Toxicomanía. Revista de Psicoanálisis, Psicoterapia y Salud Mental, 1(4), 1-21.

Hoyos Botero , C. (2000). Un modelo para investigación documental. Guía teórico-práctica sobre construcción de Estados del Arte con importantes reflexiones sobre la investigación. Medellin: HOYOS BOTERO, Consuelo (2000).

Ingelmo, J. E. (2000). El enfoque Modular-Transformacional de la psicopatologia: su aplicación al problema de la dependencia de drogas. Aperturas psicoanaliticas, 1,23.

Isella, S. (2002). Tratamiento en drogodependencias. Madrid: 1-64.

Johnson, B. (1999). Tres puntos de vista sobre la adicción. Journal of the American Phychoanalytuc Association., 47(3).

Khanthian, E. J. (1997)). The self-medication hypothesis of substance usedisorders: A reconsideration and recent applications. Harvard Review of Psychiatry, 231-244.

Kirchner, T., Torres, M., \& Forns, M. (1998). Evaluación psicologica: modelos y técnicas. Paidos, 10-45.

Lacan, J. (1989a). La dirección de la cura (T. Segovia, Trad.). En Escritos 2. México: Siglo Veintiuno Editores. Mexico: Siglo Veintiuno Editores.

Landau, J., Stanton, D., McCormick,, D., Garrett, J., Baciewicz, G., Shea, R., . . Wamboldt, F. (2004). Outcomes with the ARISE Approach to Engaging Reluctant Drug- and AlcoholDependent Individuals in Treatment. The American Journal. The American Journal of Drug and Alcohol Abuse, 30, 4, 711-748., 30(4), 711-748.

Le Poulichet, S. (2005). Capítulo 1. Teorías y tóxicos. B. La herencia de los "venenos del espíritu". En S. Le Poulichet, Toxicomanías y psicoanálisis (págs. 27-41). Buenos Aires: Amorrortu. 
Le Poulichet, S. (2005). De un imposible "tratamiento de la toxicomanía" a la elaboración de la transferencia. A. El terapeuta y el alquimista. En S. Le Poulichet, Toxicomanias y psicoanálisis. La narcosis del deseo (págs. 147-170). Buenos Aires: Amorrortu.

Lewis, H. (1987). Shame and the narcissistic personality. En Guilford. New York: D. Nathanson .

Lloyd, J., Ricketts, E., Strathdee, S., Cornelius, L., Huettner, S., Havens, J., \& Latkin, C. (2005). Social Contextual Factors Associated with Entry into Opiate.

López, C. (2006). La Adicción a Sustancias Químicas: ¿Puede ser Efectivo un Abordaje Psicoanalitico? PSYKHE, XV(I), 67-77.

López, C. (2009). La decisión de entrar a un tratamiento de adicciones: motivación propia e influencia de terceros. terapia psicológica, 27(1), 119-127.

Marín, O. D. (Diciembre de 2011). La Psicología Dinámica en el Tratamiento de la Farmacodependencia: Conceptualización y Metodología en Consulta Privada e Institucional. Poiésis, 22, 1-20.

Miller, J. A. (1997). Introducción al método psicoanalítico. Buenos Aires: Editorial : Paidós.

Moragues Bolado, T. (1999). Caso Clinico: Toxicomania un abordaje psicoanalítico. Psiquiatría pública, 11(3), 105-107.

Olivera, C. (2011). La Eficacia de la Psicoteria Psicodinámica en el Tratamiento de las Drogodepencias. Memorias del III Congreso Internacional deInvestigación y Práctica Profesional en Psicología (págs. 171-174). Buenos Aires: Ediciones de la Facultad de Psicologia - Universidad de Buenos Aires.

Palma , C. (2007). La sociedad de los a-dictos. Desde el Jardin de freud, 219-234.

Pedrero pérez, E. J., \& Et., al. (2008). Guía Clínica de Intervención Psicológica en Adicciones. Guías Clínicas Socidrogalcohol Basadas En La Evidencia Científica. Barcelona: SOCIDROGAALCOHOL Sociedad Cientifica Española de Estudios sobre el Alcohol, el Alcoholismo y otras Toxicomanías.

Renjifo, F. (2007). Una dificultad enla clinica de la toxiconanía. Desde el Jardin de freud, 95-108. .

Ressia, I. D. (2011). Dificultades en el Diágnostico y en el Tratamiento de pacientes que consumen drogas. Memorias III Congreso Internacional de Investigación y Práctica Profesional en Psicología (págs. 201-204). Buenos Aires: Ediciones de la Facultad de Psicología - Universidad de Buenos Aires.

Rojas, A. D., Ceballos, C., \& Gaspard, J. L. (2007). Actualidad de una clínica del a-sujeto. El ejemplo de la toxicomanía. Desde el jardín de Freud., 137-150. 
Sarria F., M. d. (Septiembre de 2012). Clínica de la Toxicomanía: Análisis de su Dimensión Subjetiva y Social . Trabajo de Grado de la Especialización en Psicología Clínica con Orientación Psicoanalitica., 1-24. (M. A. Moreno, Ed.) Cali., Colombia.

Shedler, J. (Febrero-Marzo de 2010). La Eficacia de la Psicoterapia Dinámica. University of Colorado Denver School of Medicine, 65(2), 98-109.

Tarrab, M. (1993). Mírenlos cómo gozan. En E. Sinatra, D. Silliti Daniel, \& M. Tarrab , En Sujeto, Goce y Modernidad, Tomo I: los fundamentos de la clinica, Tomos I y II (págs. los fundamentos de la clínica, Tomos I y II). Buenos Aires: Atuel.

Tatarsky, A. (2003). Harm reduction: Extending the reach of traditional substance use treatment. Journal of Substance Abuse Treatment, 49-256.

Velosa, J. (2009). Las toxicomanías: Algunas consideraciones críticas sobre cómo se ha comprendido el fenómeno, se han diseñado las políticas y los tratamientos. tesis Psicológica 4, 102-115.

Wurmser, L. (1984). "The role of superego conflicts in sustance abuse and their treatment. Int. J. Psychoanal. Psyhcotherapy, 10: 227- 258.

\section{Nota:}

Apoyaron la realización de este trabajo, a modo de Auxiliares de Investigación: Ana milena Ocampo Pulgarin: Psicóloga egresado Universidad de san Buenaventura. Contacto: milena_ocampo@hotmail.com. Y Camilo Alzate Jaramillo: Psicólogo egresado Universidad de san Buenaventura. Contacto: camilonalzate@hotmail.com 